\title{
MIQP-based Layout Design for Building Interiors
}

\author{
Wenming $\mathrm{Wu}^{1,2} \quad$ Lubin Fan $^{2} \quad$ Ligang Liu $^{1} \quad$ Peter Wonka ${ }^{2}$ \\ ${ }^{1}$ University of Science and Technology of China, China \\ ${ }^{2}$ King Abdullah University of Science \& Technology, Saudi Arabia
}

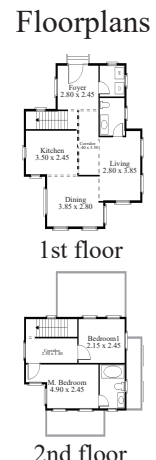

3D Rendering

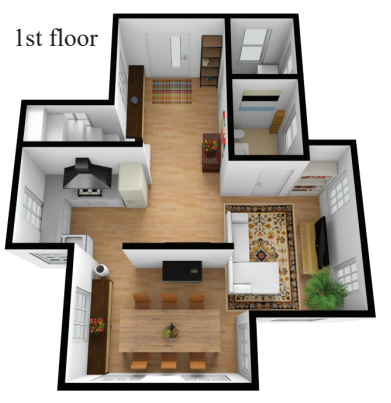

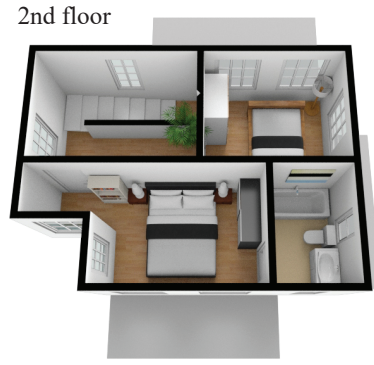

(a)
Floorplan

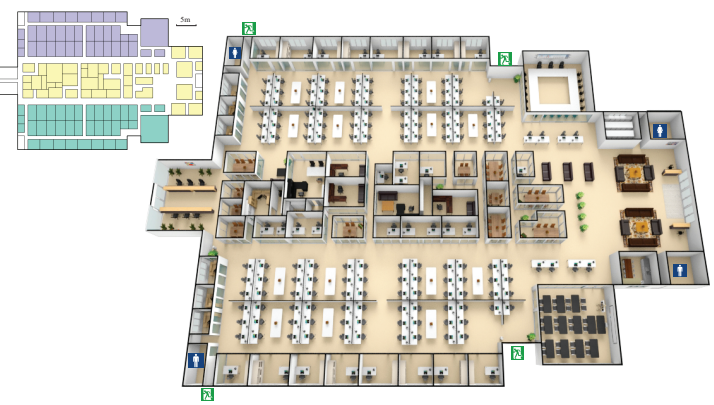

(b)

Figure 1: We propose a framework that generates building interiors with high-level constraints, e.g., room size, room position, room adjacency, and the outline of the building. (a) The layout of a two-storey house and corresponding $3 D$ renderings. (b) A large-scale example depicting an office building. For such large-scale layouts, our method is faster by multiple orders of magnitude than previous methods.

\begin{abstract}
We propose a hierarchical framework for the generation of building interiors. Our solution is based on a mixed integer quadratic programming (MIQP) formulation. We parametrize a layout by polygons that are further decomposed into small rectangles. We identify important high-level constraints, such as room size, room position, room adjacency, and the outline of the building, and formulate them in a way that is compatible with MIQP and the problem parametrization. We also propose a hierarchical framework to improve the scalability of the approach. We demonstrate that our algorithm can be used for residential building layouts and can be scaled up to large layouts such as office buildings, shopping malls, and supermarkets. We show that our method is faster by multiple orders of magnitude than previous methods.
\end{abstract}

Categories and Subject Descriptors (according to ACM CCS): I.3.5 [Computing Methodologies]: Computer GraphicsComputational Geometry and Object Modeling

\section{Introduction}

We study the automatic computation of interior building layouts, to either more quickly explore design variations for architects or to completely replace the manual modeling process for creating largescale virtual worlds. The design process typically starts by collecting a list of requirements (constraints) for the layout, e.g., room size and room adjacency. Then the designer uses trial-and-error to generate one or multiple layouts using a combination of intuition, prior experience, and professional knowledge. This usually takes from a couple of days to several weeks.
There are already several successful methods for generating building layouts automatically. The first aspect of layout generation is the determination of what constraints to use. Merrel et al. [MSK10] sample constraints from a Bayesian network. The second aspect of layout generation is the actual optimization step that computes a layout that fulfills the given constraints. Our work focuses on this part of layout generation. The most popular approach for this step is to use stochastic sampling on a framework that evaluates constraints as a black box. For example, Metropolis-Hastings (used by Merrel et al. [MSK10]), simulated annealing, or genetic algorithms are possible alternatives. The popularity of the approach 


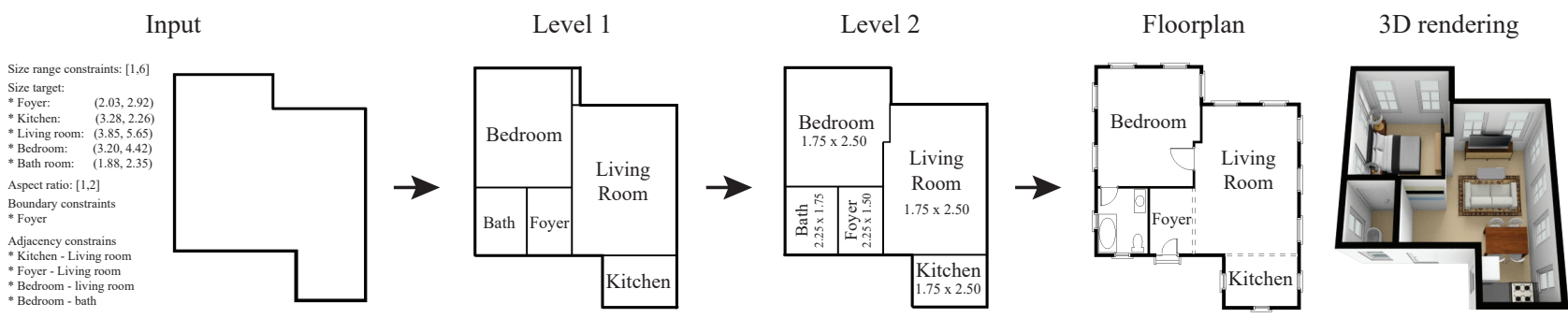

Figure 2: Overview of our framework. Given a list of high-level constraints and an outline as input (Input), we compute a layout using a hierarchical framework. Level 1 shows an initial layout and Level 2 shows the layout including local refinements. Finally, additional details can be added to visualize the results as architectural floorplans or 3D models.

is due to its generality as almost any type of constraints can be incorporated as a black box. The downside of this approach is that it degenerates on medium and larger-scale layouts and typically cannot find a single valid solution in a reasonable amount of time. We therefore propose to employ more powerful optimization techniques that scale better to larger layouts. For example, Peng et al. [PYW14] apply linear integer programming to generate layouts by tiling templates. Their tilling-based approach scales better, but it is less general. They can use only pre-defined room templates.

Our new framework overcomes the scalability problem of stochastic methods without all the restrictions on generality imposed by the tiling-based approach. Our solution has multiple components that are coordinated with each other. We formulate the layout optimization problem as a mixed integer quadratic programming (MIQP) problem. The integer variables are used to account for different room configurations. An integral component to this formulation is our proposed parametric layout representation. Each room is represented as a polygon that consists of a set of small rectangles. This enables us to generate rooms with various shapes in contrast to Peng et al. [PYW14]. In addition, this representation enables us to formulate important high-level constraints about room size and adjacency in a manner that is compatible with MIQP. We also propose a hierarchical framework to improve the scalability of our approach. Fig. 1 shows layouts of a residential building and an office building generated by our algorithm.

We demonstrate our method on small-scale layouts of residential buildings and large-scale layouts such as office buildings and supermarkets. Our main contributions are as follows:

- We propose a layout computation method that is faster by multiple orders of magnitude than previous work built on stochastic optimization.

- We propose a parametric representation of interior layouts that is more general than previous methods based on tiling.

- We can generate results on a larger scale than achieved by previous work.

\section{Related Work}

Our work relates to research in three areas: VLSI design, architectural space planning, and urban layout modeling.

VLSI design. VLSI designs can be categorized as slicing struc- tures that are presented by binary trees, and non-slicing structures that are more general. Topological representations are crucial for VLSI floorplan design. Popular VLSI floorplan representations include the following: sequence pair (SP) [SVW*11], bounded slicing grid (BSG) [NFMK97], $\mathrm{B}^{*}$-tree [MXM09], ordered tree (OTree) [NNA06], transitive closure graph (TCG) [LC04], corner block list (CBL) [DZH $\left.{ }^{*} 02\right]$, and integer coding (IC) [CGC10]. Most VLSI design algorithms search for a floorplan using stochastic approaches, e.g., simulated annealing [Sec12], genetic algorithms [SDD12], particle swarm optimization [CGC10], and differential evolution [MAR07]. Simulated annealing is popular in modern design problems using the $\mathrm{B}^{*}$-tree $[\mathrm{CC} 06]$ and sequence pair [KF00], but it requires expensive computations and it can struggle to achieve optimal or near optimal solutions. Genetic algorithms [CZ10, SDD12] are another algorithm class explored by researchers in their quest for good floorplan algorithms. However, as a generate-and-test methodology, genetic algorithms have very similar shortcomings to simulated annealing. We propose a more general representation for layout design in which each room is represented as a polygon that can be further decomposed into a set of rectangles and our constraints are different.

Architectural space planning. Architectural space planning is concerned with planning buildings of different scales. One popular approach is to apply an expert system [FUC $\left.{ }^{*} 88, \mathrm{KS} 05\right]$. RíoCidoncha et al. [DRCMPI07] develop a model for facility layout design by combining an expert system and artificial intelligence. Another class of methods applies shape grammars that consist of a set of rules that is recursively applied to an initial assertion to produce a final statement, e.g., [RCMLS96, LHP11]. Harada et al. [HWB95] develop a system for interactive manipulation of architectural layouts by using shape grammars. Duarte [Dua05] proposes an interactive system for generating layouts on the web based on a discursive grammar that consists of a programming grammar and a designing grammar. The evolutionary approach is also used in architectural space planning [EF99, Nil06, Dou07]. Bahrehmand et al. [BBM*17] present an interactive layout solver based on the evolutionary approach. It assists designers in layout planning by recommending personalized space arrangements according to architectural guidelines and user preferences. The major drawback of evolutionary algorithms is the poor performance. The constraintbased approach [BF91,LFT00,Hsu00,DB08] seems to be a promising direction for our work. 
Merrel et al. [MSK10] learn attributes of rooms from a given dataset by a Bayesian network and synthesize the layout by the stochastic method. The main problem of the approach is that it is not suitable for large-scale examples due to the stochastic method. Rosser et al. [RSM17] also propose a data-driven method. They learn building measurements to produce interior plans. In [LYAM13], the authors present an interactive system to create interior room layouts subject to design constraints, user preferences, and manufacturing considerations. Hua et al. [Hua16] develop a method for automatically constructing irregular floor plans. Given image patterns, the program produces a variety of layouts that satisfy both geometric and topological requirements. Bao et al. [BYMW] propose a method to explore building layouts. To obtain variations in connectivity, they use standard simulated annealing. Liu et al. [LWKF17] apply integer programming to encode high-level constraints. However, their goal is to evaluate the extracted constraints in recovering the floorplan data from a raster image.

Urban layout modeling. Our problem also relates to urban layout modeling. [Ali12, STBB14] review the topic in a broad context. One common approach generates large-scale layouts by first creating the road network and then by generating parcels. An interactive example-based system for synthesizing urban layouts is proposed by [AVB08]. They use the structure and image data of real-world urban areas for complex urban layout generation. Network design can be seen as a dual problem in layout design. Chen et al. [CEW*08] create road networks from an existing street network by designing an underlying tensor field and editing the graph representation. Yang et al. [YWVW13] combine hierarchical splitting and global optimization to guarantee the fairness and regularity of the generated streets and parcels. Improving upon this previous work, Peng et al. [PYW14] propose a novel tiling-based approach with deformable templates. Their subsequent work [PYB* 16] proposes an integer-programming-based approach to generate networks starting from functional specifications. Feng et al. [FYY*16] focus on mid-scale layout modeling based on human crowds. Given a layout domain, their approach synthesizes crowd-aware layouts by considering the crowd flow. Urban layout modeling has also been widely used in the design of video game environments. Ma et al. [MVLS14] propose an algorithmic approach for automatically laying out complex game levels that conform to the designer's specifications.

\section{Overview}

Our framework consists of the following components:

- First, we introduce a parametric representation that describes a layout by a set of axis-aligned polygons. To make the model better suitable for optimization, we also present a rectangle-based layout representation (Section 4).

- Second, we describe the basic formulation of our MIQP-based method (Section 5.1).

- Third, we describe a set of high-level constraints for layout generation (Section 5.2).

- Fourth, we propose a hierarchical framework to extend the basic method (Section 5.3).
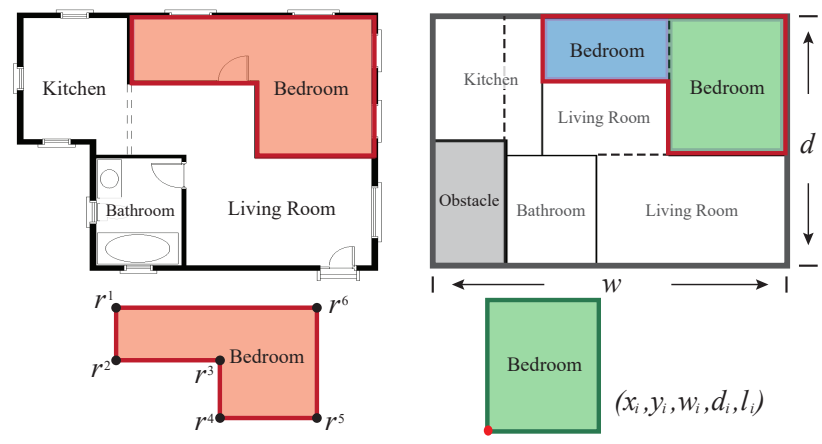

Figure 3: An interior layout consists of four rooms, i.e., living room, bedroom, bathroom, and kitchen. Left: each room is represented as an axis-aligned polygon defined by points, $r=\left\{r^{i}\right\}$. Right: the layout is presented by a set of room rectangles by decomposing the polygonal room into small rectangles with the same label. A room rectangle is described by a tuple $\left(x_{i}, y_{i}, w_{i}, d_{i}, l_{i}\right)$. The layout domain is presented as the bounding box of the domain subtracted by obstacle rooms shown in gray.

- Fifth, we evaluate our method and perform comparisons with previous methods on examples of different scales (Section 6).

Fig. 2 shows an example of generating a layout of an apartment. Given the outline of the apartment and a set of user-specified highlevel constraints, our method generates the layout in a coarse-tofine manner, i.e., two levels in this example. Fig. 2 right shows the final result.

\section{A Parametric Layout Representation}

Layout $L$ of a building consists of its domain $D$ given as an axisaligned polygon and rooms $R=\left\{r_{i}\right\}_{i=1}^{N}$, where $N$ is the number of rooms in the layout (see Fig. 3 left). The shape of the domain is an axis-aligned polygon that is defined by an array of vertices, $D=\left\{d_{i}\right\}$. Each room is also an axis-aligned polygon defined by vertices, $r_{i}=\left\{r_{i}^{j}\right\}$, and a label, $l$, to describe its functionality, e.g., kitchen, living room, etc. Given the layout domain, the goal of the layout design problem is to arrange a set of rooms inside the domain according to some constraints. A valid layout should satisfy two basic constraints: first, all rooms are inside the domain; second, there is no overlap between rooms. A layout should also satisfy a set of high-level constraints describing how a synthesized layout should behave, e.g., room size and adjacency.

There are two challenges of this parametric representation. First, the number of vertices of each polygonal room is not known in advance. This means that the number of variables has to change during the optimization, even with a fixed number of rooms. Second, it is not easy to formulate high-level constraints for arbitrary polygons. For example, expressing constraints about the adjacency between general polygonal rooms is very difficult. We therefore introduce the computational object, room rectangle, that is defined as the basic element of a layout (see Fig. 3 right). A room rectangle is described by a tuple $\left(x_{i}, y_{i}, w_{i}, d_{i}, l_{i}\right)$ where $\left(x_{i}, y_{i}\right)$ denotes the position of the bottom-left corner of the rectangle, $\left(w_{i}, d_{i}\right)$ denotes the 


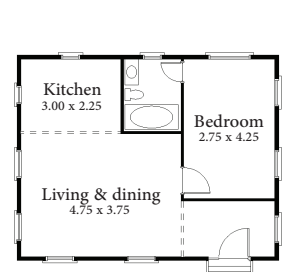

(a)

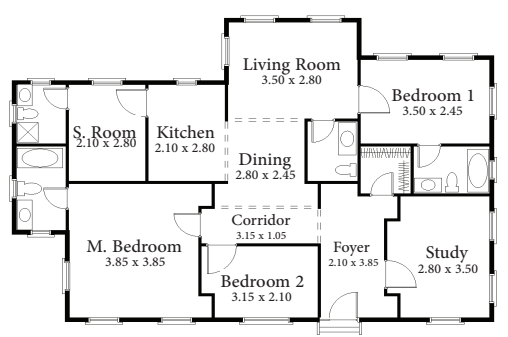

(b)
Figure 4: Layouts synthesized by our algorithm. Left: the basic formulation can produce layouts in which both the domain and rooms are rectangles. Right: a complicated layout synthesized by the hierarchical framework with user-specified high-level constraints.

width and depth, and $l_{i}$ denotes its label. Then, a polygonal room, $r_{i}$, can be presented as the union of a set of room rectangles with the same label. The boundary of the room polygon is the outline of the union of rectangles in the polygon. The layout domain can also be presented by rectangles (see Fig. 3). The polygonal domain can be described as its bounding box minus a set of special rectangles labeled as obstacle. Such obstacle rectangles cannot be covered by any room. By introducing the room rectangle, the layout, $L$, can be presented by a set of rectangular rooms. Given the number of room rectangles of the layout, $N$, the number of parameters is fixed, i.e., $4 \times N$. Moreover, the relationship between rectangles is easy to evaluate. According to the definitions of the interior layout and the room rectangle, our problem is to find the optimal set of rectangles, i.e., $\left\{\left(x_{i}, y_{i}, w_{i}, d_{i}, l_{i}\right)\right\}$.

Given the layout domain and the number of rooms, the interior layout design task changes to generate a valid layout in which room rectangles are tiled in the domain with optimal parameters. We apply mixed integer quadratic programming (MIQP) optimization to solve the tiling problem. Then, we obtain the final layout by merging connected room rectangles with the same label.

\section{MIQP-based Hierarchical Layout Design}

We introduce our algorithm starting from the simplest case in which both the layout domain and the rooms are rectangles (Fig. 4 left). Next, we introduce five high-level constraints abstracted from real floorplans to generate more realistic layouts. Finally, we introduce a hierarchical framework for more complicated layout generation in which the layout domain and rooms are polygons (Fig. 4 right).

\subsection{Basic Formulation}

Our goal is to create a valid layout that covers the whole domain. We consider the layout to be valid if it satisfies two basic constraints: the inside constraint, which ensures that all rooms are inside the boundary, and the non-overlap constraint, which ensures that there is no overlap between rooms.

Inside constraint, $C_{\text {inside }}$. In a valid layout, we require that all rooms are inside the layout domain. Since the domain and rooms are all rectangles, the constraint requires that four corners of each rectangle are inside of the bounding box of the domain. The constraint is expressed as

$$
\left\{\begin{aligned}
0 \leq x_{i} & \leq w \\
0 \leq y_{i} & \leq d \\
x_{i}+w_{i} & \leq w \\
y_{i}+d_{i} & \leq d,
\end{aligned}\right.
$$

where $1 \leq i \leq N, N$ is the number of rooms, and $w$ and $d$ are the width and depth of the bounding box of the domain, respectively. For the case of a polygonal domain, we update the inside constraint in Section 5.3.

Non-overlap constraint, $C_{\text {overlap }}$. We require that there is no overlap between any pair of rooms. There are four kinds of relationship between two rectangular rooms, i.e., room $i$ is on the front/back/left/right side of room $j$. The difficulty of setting this constraint is that we have no prior knowledge of which case it is. We introduce an auxiliary binary variable, $\sigma_{i, j}^{d}$, into our problem for each pair of rooms $(i, j)$ to select the relationship automatically, where $d \in\{$ front, back,left, right $\}$, and $\sigma_{i, j}^{d}=1$ means that the relationship $d$ of room $i$ and room $j$ is selected. The non-overlap constraint is then defined as

$$
\left\{\begin{aligned}
x_{i}-w_{j} & \geq x_{j}-M \cdot\left(1-\sigma_{i, j}^{R}\right) \\
x_{i}+w_{i} & \leq x_{j}+M \cdot\left(1-\sigma_{i, j}^{L}\right) \\
y_{i}-d_{j} & \geq y_{j}-M \cdot\left(1-\sigma_{i, j}^{F}\right) \\
y_{i}+d_{i} & \leq y_{j}+M \cdot\left(1-\sigma_{i, j}^{B}\right) \\
\sum_{d=1}^{4} \sigma_{i, j}^{d} & \geq 1,
\end{aligned}\right.
$$

where $M$ is a large constant to ensure that there is no overlap between room $i$ and room $j$ in direction $d$ when $\sigma_{i, j}^{d}=1$, and the inequality is always established when $\sigma_{i, j}^{d}=0$. In our implementation, we set $M=w+d$. The last inequality ensures that at least one of the above cases should be satisfied. Note that the introduced auxiliary binary variables, $\sigma_{i, j}^{d}$, encode the relationship between room $i$ and room $j$ in the constraint, and they are assigned automatically during the optimization. This method is also used in the following constraints.

Objective function. We evaluate valid layouts that satisfy the two basic constraints, $C_{\text {inside }}$ and $C_{\text {overlap }}$, based on an energy function that prefers layouts that cover the domain as much as possible. The energy function is defined as

$$
E_{\text {cover }}(L)=\operatorname{Area}(L)-\sum_{i} w_{i} \times d_{i},
$$

where $\operatorname{Area}(\cdot)$ is the area operator. Minimizing Eq. 3 under the two basic constraints, $C_{\text {inside }}$ and $C_{\text {overlap }}$, yields a mixed integer program with a quadratic objective function (MIQP) and linear constraints,

$$
\min _{L} E_{\text {cover }}(L) \text {. }
$$

Fig. 4 left shows a layout generated by the basic formulation.

\subsection{Optional High-level Constraints}

We model five optional high-level constraints that are essential to interior building layout generation.

Size control. The size of each room can be controlled by two 
approaches: adding size range constraints for rooms and specifying target sizes for rooms. The range of the room size can be constrained by providing the minimum and maximum values of $w_{i}$ and $d_{i}$. Then, the size range constraint, $C_{\text {size }}$, is defined as

$$
\left\{\begin{array}{l}
\underline{w}_{i} \leq w_{i} \leq \bar{w}_{i} \\
\underline{d}_{i} \leq d_{i} \leq \bar{d}_{i}
\end{array}\right.
$$

where $\left(\underline{w}_{i}, \underline{d}_{i}\right)$ and $\left(\bar{w}_{i}, \bar{d}_{i}\right)$ are the minimum and maximum sizes of room $i$, respectively. The user can also specify target sizes for rooms. Then, the size error of rooms can be evaluated by a size error term, which is defined as

$$
E_{\text {size }}=\sum_{i}\left(w_{i}-w_{i}^{*}\right)^{2}+\left(d_{i}-d_{i}^{*}\right)^{2},
$$

where $\left(w_{i}^{*}, d_{i}^{*}\right)$ is the target size of room $i$. We can encourage room sizes to become close to the user-specified values by adding $E_{\text {size }}$ to the objective function.

Aspect ratio constraint, $C_{\text {ratio }}$. We provide a room aspect ratio constraint, $C_{\text {ratio }}$, to avoid generating rooms that are too wide or too narrow. Similar to $C_{\text {overlap }}$, we add an auxiliary binary variable for each room to adjust the orientation (i.e., horizontal and vertical) of the room rectangle. The aspect ratio constraint is defined as

$$
\left\{\begin{aligned}
& \underline{r}_{i} \cdot d_{i} \leq w_{i}+M \cdot \sigma_{i} \\
& \bar{r}_{i} \cdot d_{i} \geq w_{i}-M \cdot \sigma_{i} \\
& \underline{r}_{i} \cdot w_{i} \leq d_{i}+M \cdot\left(1-\sigma_{i}\right) \\
& \bar{r}_{i} \cdot w_{i} \geq d_{i}-M \cdot\left(1-\sigma_{i}\right),
\end{aligned}\right.
$$

where $\left(\underline{r}_{i}, \bar{r}_{i}\right)$ are the minimum and maximum aspect ratios between $w_{i}$ and $d_{i}$ of room $i$, and $\underline{r}_{i} \geq 1 . \sigma_{i}=0$ means that room $i$ is a horizontal room and its aspect ratio is in the range of $\left[\underline{r}_{i}, \bar{r}_{i}\right] . \sigma_{i}=1$ means that room $i$ is a vertical room.

Position constraint, $C_{p o s}$. During the design process, sometimes the designer has to specify the approximate position for a room, e.g., the foyer should connect to the main door. A guide position for a room is presented as a single point $\left(x^{*}, y^{*}\right)$ or two points. For each point, the position constraint requires the room to cover the point:

$$
\left\{\begin{array}{l}
x_{i} \leq x^{*} \leq x_{i}+w_{i} \\
y_{i} \leq y^{*} \leq y_{i}+d_{i}
\end{array}\right.
$$

Boundary constraint, $C_{\text {boundary }}$. Sun exposure is an important planning criterion. For example, people typically have a preference between the master bedroom receiving light in the morning (facing east) or not receiving light in the morning (facing west). To fulfill these types of constraints, we add a boundary constraint that requires the room to be adjacent to at least one of a set of userspecified edges of the domain polygon. We introduce a binary variable, $\rho_{k}(k=1,2, . ., n)$, for edge $k$ where $n$ is the number of edges of the domain polygon, and $\rho_{k}=1$ indicates that the room is adjacent to edge $k$. For each candidate edge in the given set, we formulate the constraint to check if the edge covers the corresponding edge of the room. Suppose that the edge is a bottom edge. It is then defined as $\left[\left(x_{1}, y\right),\left(x_{2}, y\right)\right]$ (i.e., a horizontal edge on the bottom side of the domain polygon), Fig. 5 left illustrates the constraint for room $i$,
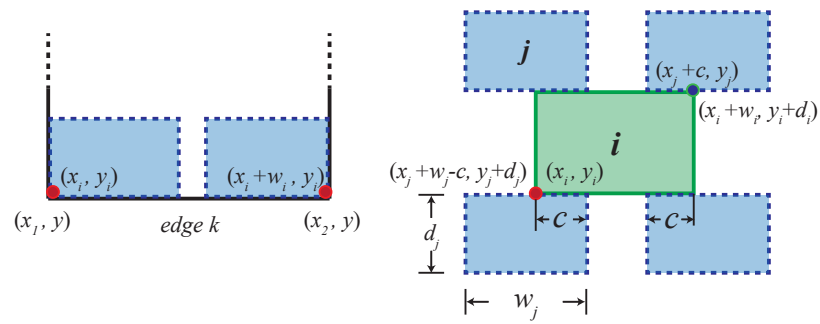

Boundary constraint

Adjacency constraint

Figure 5: Left: an example of a boundary constraint that requires the room to be adjacent to the bottom edge, $k$, of the layout domain (black). Two extreme positions for room i (blue) are shown. Right: an example of an adjacency constraint that requires room $i$ (green) and room $j$ (blue) to be vertically connected. Four extreme positions for room $j$ are shown. To ensure sufficient connection, we require the minimum length of the common edge between two connected rooms to be $c$.

and it is expressed as

$$
\left\{\begin{aligned}
y_{i} & \leq y+M \cdot\left(1-\rho_{k}\right) \\
x_{i} & \leq x_{2}-w_{i}+M \cdot\left(1-\rho_{k}\right) \\
x_{i} & \geq x_{1}-M \cdot\left(1-\rho_{k}\right) \\
\sum_{k=1} \rho_{k} & \geq 1,
\end{aligned}\right.
$$

where the last inequality signifies that at least one of the candidate edges should be selected. There are two differences between $C_{\text {boundary }}$ and $C_{\text {pos }}$. First, $C_{\text {pos }}$ requires the room to cover the specified positions, while $C_{\text {boundary }}$ requires the room to select one edge from a candidate set. Second, the candidate edge is always on the boundary of the layout domain, but users can specify any position for constraint, $C_{p o s}$.

Adjacency constraint, $C_{a d j}$. The user can also specify two rooms to be adjacent, e.g., the master bedroom is connected to the living room. Suppose that room $i\left(x_{i}, y_{i}, w_{i}, d_{i}\right)$ and room $j$ $\left(x_{j}, y_{j}, w_{j}, d_{j}\right)$ should be connected. The idea for modeling this constraint is to force the two rooms to overlap. In combination with the non-overlap constraint, $C_{\text {overlap }}$, that forbids the inside of the rooms to overlap, this will force the overlap to happen only at the boundary of the rooms. This is not sufficient, however, because we would also like to ensure that there is a minimum overlap, $c$, called the contact length, of the common edge between the two connected rooms. The contact length is important to ensure that there is enough space for adding a door between the two rooms. The constraint can be written as

$$
\left\{\begin{aligned}
x_{i} & \leq x_{j}+w_{j}-c \cdot \theta_{i, j} \\
x_{i}+w_{i} & \geq x_{j}+c \cdot \theta_{i, j} \\
y_{i} & \leq y_{j}+d_{j}-c \cdot\left(1-\theta_{i, j}\right) \\
y_{i}+d_{i} & \geq y_{j}+c \cdot\left(1-\theta_{i, j}\right),
\end{aligned}\right.
$$

where $\theta_{i, j}$ is a binary variable that determines the connection direction automatically. $\theta_{i, j}=1$ denotes a vertical connection and otherwise a horizontal connection. Fig. 5 right illustrates the constraint for a vertical connection. We extend the constraint to allow room $i$ to connect either room $j$, room $k$ or both. Then the constraint 


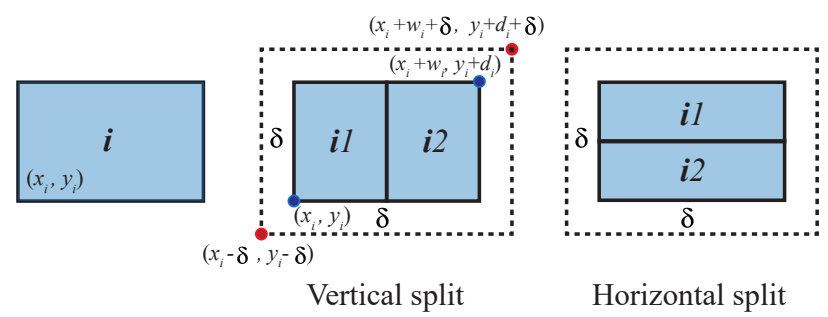

Figure 6: Room decomposition in a hierarchical framework. Given a rectangular room in the sub-domain, we decompose it into two small rectangular rooms with the same size in a random direction, i.e., a vertical split or a horizontal split.

is expressed as

$$
\left\{\begin{aligned}
x_{i}+w_{i} & \geq x_{j}+c \cdot \theta_{i, j}-M \cdot \sigma_{i, j, k} \\
x_{i} & \leq x_{j}+w_{j}-c \cdot \theta_{i, j}+M \cdot \sigma_{i, j, k} \\
y_{i} & \leq y_{j}+d_{j}-c \cdot\left(1-\theta_{i, j}\right)+M \cdot \sigma_{i, j, k} \\
y_{i}+d_{i} & \geq y_{j}+c \cdot\left(1-\theta_{i, j}\right)-M \cdot \sigma_{i, j, k} \\
x_{i}+w_{i} & \geq x_{k}+c \cdot \theta_{i, k}-M \cdot\left(1-\sigma_{i, j, k}\right) \\
x_{i} & \leq x_{k}+w_{k}-c \cdot \theta_{i, k}+M \cdot\left(1-\sigma_{i, j, k}\right) \\
y_{i} & \leq y_{k}+d_{k}-c \cdot\left(1-\theta_{i, k}\right)+M \cdot\left(1-\sigma_{i, j, k}\right) \\
y_{i}+d_{i} & \geq y_{k}+c \cdot\left(1-\theta_{i, k}\right)-M \cdot\left(1-\sigma_{i, j, k}\right)
\end{aligned}\right.
$$

where $\sigma_{i, j, k}$ is a binary variable, $\sigma_{i, j, k}=0$ means that room $i$ is connected to room $j$; otherwise room $i$ is connected to room $k$.

Extended objective function. The final IP problem is defined as

$$
\min _{L, \sigma, \theta, \rho} \lambda_{\text {cover }} E_{\text {cover }}(L)+\lambda_{\text {size }} E_{\text {size }}(L),
$$

subject to the set of linear constraints expressed above, $L=$ $\left\{\left(x_{i}, y_{i}, w_{i}, d_{i}\right)\right\}$ are rectangular room tuples. $\sigma, \theta$, and $\rho$ are auxiliary binary variables selected automatically. $\lambda_{\text {cover }}$ and $\lambda_{\text {size }}$ are weights that control the trade-off between the coverage term, $E_{\text {cover }}$, and the size error term, $E_{\text {size }}$. In our implementation, we set $\lambda_{\text {cover }}=\lambda_{\text {size }}=1$. Please note that since both the coverage term, $E_{\text {cover }}$, and the size error term, $E_{\text {size }}$, are quadratic terms, Eq. 12 is a mixed integer quadratic problem.

\subsection{Hierarchical Framework}

In this section, we propose a hierarchical framework to extend the basic method to improve the details of each polygonal room and to generate large-scale layouts efficiently. There are five main components for layout generation in each level. Fig. 7 shows the pipeline of our hierarchical framework.

Polygonal layout domain representation. We describe a polygonal layout domain by a set of rectangles, i.e., by the bounding box subtracted by special rectangles labeled as obstacle, which means that they cannot be covered by any room. All rectangular rooms labeled as obstacle are retained during the following process.

Sub-domain selection. Once we obtain a layout, we can select a sub-domain for further refinement. A sub-domain is also a layout domain that is presented as a polygon, and it consists of a set of rectangular rooms generated during the previous optimization.
The sub-domain is selected automatically by the following strategies. First, if the layout domain is not completely covered by rooms generated in the previous layout, then we select the polygon with rectangular rooms that surround the empty region. Second, if the size error of a rectangular room is larger than a threshold, then we select the polygon with that room and its neighboring rooms as the sub-domain. Moreover, the sub-domain can also be specified by the user.

Initialization by room decomposition. We initialize the layout optimization by decomposing rooms in the sub-domain. Given a rectangular room in the sub-domain, we decompose it into two small rectangular rooms of the same size in a random direction (see Fig. 6). Each small rectangular room inherits the label from its parent.

Constraints update. Given the new layout domain and initialized rooms inside the domain, we set the constraints for the new MIQP optimization as follows. First, we update the inside constraint, $C_{\text {inside }}$, and the non-overlap constraints, $C_{\text {overlap }}$, using the new layout domain and rectangular rooms. For a polygonal domain, the inside constraint for each room rectangle is presented by two parts: first, the rectangle should be inside the bounding box of the domain; second, the rectangle cannot cover any obstacle. Second, for the original room with position constraint, $C_{p o s}$, we set the new position constraint for its child, which has to cover the position guidance and leave the position constrain free for the other child. We update the boundary constraint, $C_{\text {boundary }}$, in the same manner. Third, an additional room adjacency constraint for two child rooms is added to keep them connected in the final layout. Fourth, we add a size refinement constraint, $C_{\text {refine }}$, for each child room (i.e., $r_{i 1}$ and $r_{i 2}$ ) to replace the size constraints on their parent room, $r_{i}$. The idea of $C_{\text {refine }}$ is that we can restrict size changes of each child room in a small refinement range, $\delta$, to avoid significant changes in the final layout. Suppose a vertical split is applied in the previous step (see Fig. 6 middle). Then, the refinement constraint is defined as

$$
\left\{\begin{aligned}
x_{i 1} & \geq x_{i}-\delta \\
x_{i 1} & \leq x_{i} \\
y_{i 1} & \geq y_{i}-\delta \\
y_{i 1} & \leq y_{i} \\
y_{i 1}+d_{i 1} & \geq y_{i}+d_{i} \\
y_{i 1}+d_{i 1} & \leq y_{i}+d_{i}+\delta \\
x_{i 2}+w_{i 2} & \geq x_{i}+w_{i} \\
x_{i 2}+w_{i 2} & \leq x_{i}+w_{i}+\delta \\
y_{i 2} & \geq y_{i}-\delta \\
y_{i 2} & \leq y_{i} \\
y_{i 2}+d_{i 2} & \geq y_{i}+d_{i} \\
y_{i 2}+d_{i 2} & \leq y_{i}+d_{i}+\delta
\end{aligned}\right.
$$

where $\left(x_{i 1}, y_{i 1}, w_{i 1}, d_{i 1}\right)$ are parameters of child room $i 1$, $\left(x_{i 2}, y_{i 2}, w_{i 2}, d_{i 2}\right)$ are parameters of child room $i 2$, and $\delta$ is the refinement range. The refinement constraint for the horizontal split is presented in the supplementary material.

Optimization. We formulate the objective function as Eq. 12 subject to the updated constraints for the sub-domain. The generated layout will replace the original rectangular rooms in the subdomain. If there are several sub-domains, we apply the same procedure to all of them sequentially. We move to the next level when 


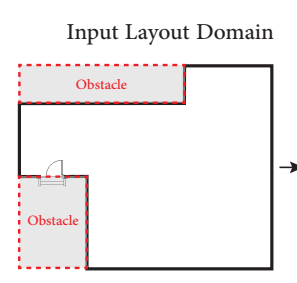

(a)

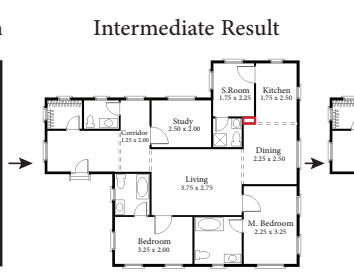

(b)

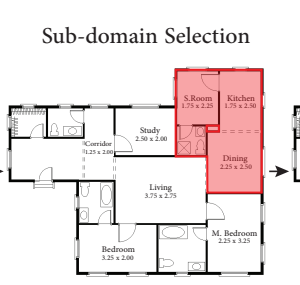

(c)

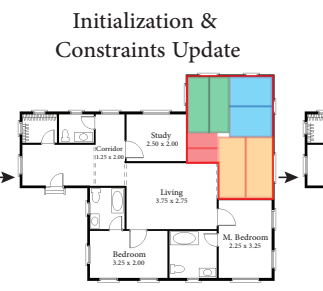

(d)

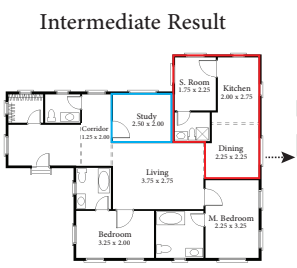

(e)
Final Result

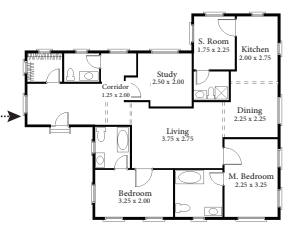

(f)

Figure 7: Pipeline of the hierarchical framework. (a) Starting from the empty layout domain with the door position and two obstacles, our method generates $(b)$ an interior layout on the first level according to the user-specified high-level constraints. There is a region (shown in red) that is not covered by any room. (c) Our algorithm automatically selects the sub-domain (highlighted as the red polygon) for the optimization on the next level. (d) Then, the sub-domain is initialized with small rectangles and constraints are updated. (e) The new layout is generated in the sub-domain (i.e.,red polygon). The user specifies the study room (blue rectangle) for further improvement. $(f)$ The final result.

all sub-domains are optimized. Starting from an empty layout domain, we generate the layout design in a coarse-to-fine manner by the proposed hierarchical framework. We terminate the hierarchical optimization when the layout domain is completely covered and the size error of each room is smaller than the threshold. To obtain the final interior layout, which is represented by polygonal rooms, we merge rectangular rooms with the same label.

Large-scale layout generation. We apply a hierarchical framework for large-scale layout generation. Fig. 8 shows an example of a shopping mall that is generated with four levels. In practice, the large-scale layouts can be decomposed into regions and rooms in each region. Our idea is to generate the layout for regions first, then generate the layout inside each region. Given the layout domain and constraints for regions, we apply the hierarchical framework to generate the layout for regions. The polygonal regions are generated in a coarse-to-fine manner by the aforementioned framework. Then, aisles are generated by expanding the gap between two regions with a fixed width if needed. Next, we set each region as a sub-domain and assign constraints for each sub-domain. The number of rooms in each sub-domain and high-level constraints for them are specified by the user. Then, we formulate the objective function for each sub-domain and generate the corresponding layouts. We repeat this procedure to get the final layout. Fig. 1(b) shows another example of a layout of an office building.

\subsection{Implementation Details}

We implement our algorithm in C++ using Gurobi [GO16] to solve the MIQP problem. As it is difficult to find a globally optimal solution, we also accept sub-optimal solutions that fulfill all constraints computed within reasonable time limits.

Users can set some of the high-level constraints on a subset of rooms or on all of them. Since modeling realistic constraints is a challenging task, we also provide an option for automatic constraint modeling for residential buildings. We collect 200 floorplans of residential buildings and learn the size constraints and adjacency constraints. We first train polynomial regression models for width and depth of each kind of room with respect to the square root of the domain area. Given a new domain, the range of the width and depth of each room are set as the range of the $90 \%$ confidence band of the corresponding model. We also collect connectivity information from the data to randomly sample a useful set of adjacency constraints. For convenience, we formulate all the constraints and objective functions as templates and design a semi-automatic system for specifying constraints. For example, a user can specify a room type and a constraint type and then our system generates all constraint details automatically. For a moderate layout (15 rooms with 50 constraints), the user usually requires 2-5 minutes to set all constraints.

The performance of synthesizing a good layout is related to the size constraints of rooms. In practice, we apply several optimization strategies for acceleration. If target sizes of rooms are given, we apply a heuristic to set up the minimum and maximum sizes for specified rooms to help Gurobi quickly find the feasible solution. We first set $90 \%$ and $110 \%$ of the target value as the minimum and maximum size, respectively. Then, we gradually relax the size of the constraints by $10 \%$ until Gurobi finds a feasible solution within reasonable time limits. For a task that has 20 rooms or less, we set the time for accepting sub-optimal solutions to 5 seconds. The time increases proportionally with the number of rooms in complicated tasks.

We also apply a heuristic to add doors and windows. A door is added between two rooms that are assigned an adjacency constraint. For a residential building, we define the door policy in advance and apply it to the generated layout. Windows are evenly distributed on the outside walls. Furniture is placed manually. Fig. 10 shows an example of generated layouts with assets and the corresponding three-dimensional model is also shown.

\section{Results and Applications}

All of our experiments are performed on a laptop with dual $2.9 \mathrm{GHz}$ Intel Core i5 processors and 8 GB main memory.

Interior layout for residential buildings. First, we apply our method to an apartment layout design. The layout domain, the number of rooms and the functionality of each room are given. In Fig. 9, we show two interior layouts designed by our algorithm with the same layout domain and constraints. Boundary constraints are 

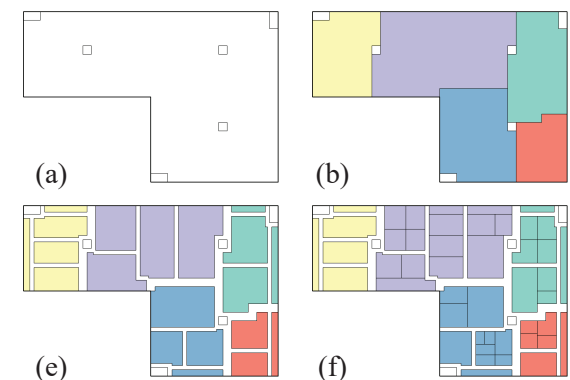

(c)
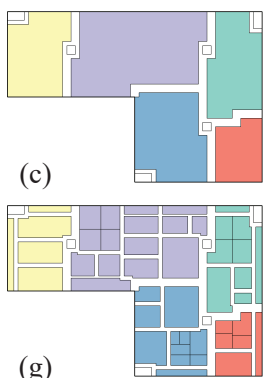

(d)
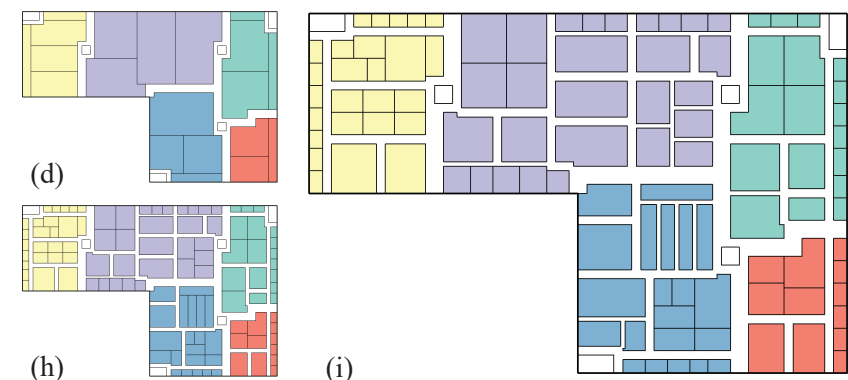

(i)

Figure 8: An interior layout of a shopping mall is generated by our hierarchical framework. (a) Starting from an empty layout domain with obstacles. (b) First, we generate the layouts for five regions shown in different colors. (c) Then aisles are generated by expanding the gap between two regions with a fixed width. (d) Next, we generate the layouts inside each region. $(e-h)$ We repeat this procedure to get (i) the final layout. The number of objects (regions and rooms) and constraints on each level are specified by the user.

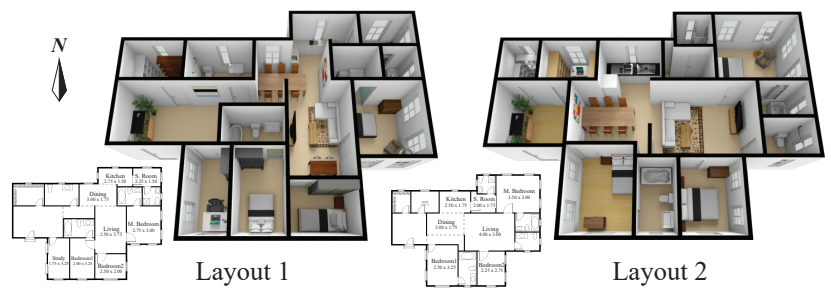

Figure 9: Two apartment layouts are generated by our algorithm with the same input. Boundary constraints are added for bedrooms to ensure that all bedrooms receive sufficient sunshine from the south east direction. The north direction is shown.

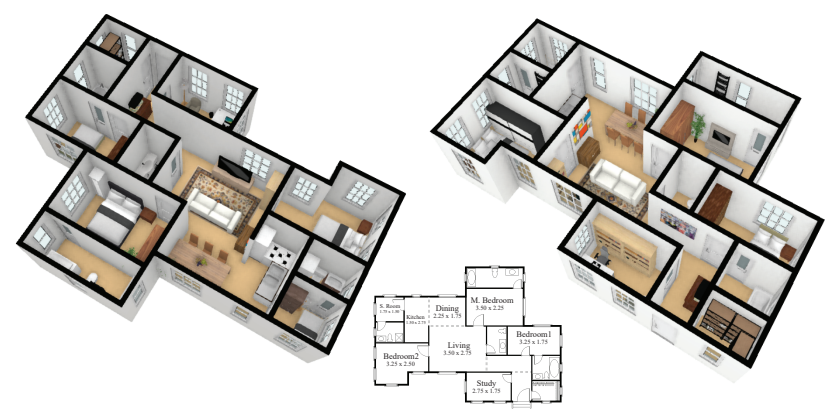

Figure 10: An interior layout of a bungalow-style house generated by our algorithm. The floorplan is shown in the middle. Two sides of the $3 D$ rendering are shown.

added for bedrooms to ensure that all bedrooms receive sufficient sunshine from the south-east direction. Fig. 10 shows a layout generated for a bungalow-style house. Our method can be extended to design layouts for a multi-storey house by taking the stairs (or elevator) as a special room that should be consistent on every floor. Fig. 11 shows the layout of a two-storey house. The supplementary materials present more results.

Evaluation of our method. First, we evaluate our approach by reproducing interior layouts of buildings. A good layout algorithm should reproduce reasonable results from constraints ex-
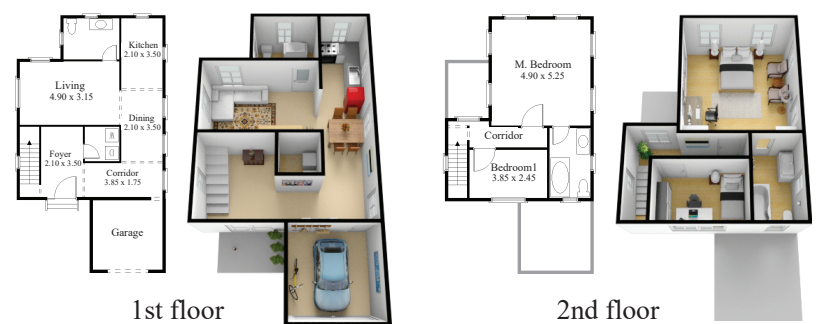

Figure 11: An interior layout of a two-storey house. The stairs are considered as special rooms that should be consistent between two floors. The floorplan of each floor is shown on the left, $3 D$ renderings are shown on the right.
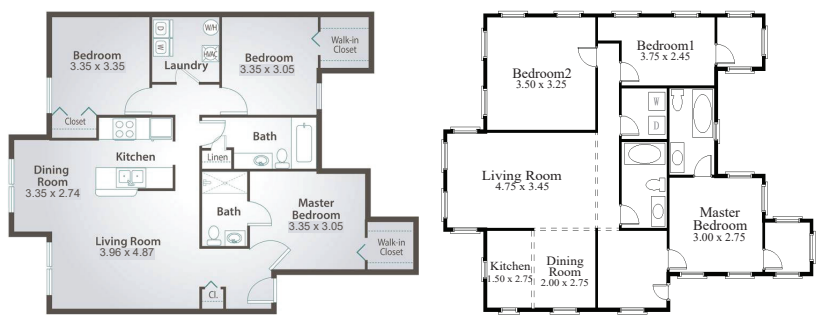

Figure 12: Regeneration of a layout according to the constraints extracted from a given layout. Left: the ground-truth layout. Right: an interior layout regenerated from the size and adjacency constraints extracted from the left layout.

tracted from real designs. We extract only size and room adjacency constraints from the given design shown in Fig. 12 left. A regenerated layout is shown in Fig. 12 right. We find that all connectivities are maintained and that the size error of each room is small. Second, we perform a comparison between layouts generated by the basic method and by our hierarchical approach with the same layout domain and high-level constraints. Since the basic approach only tiles rectangles on one level, there are some small regions remaining (see Fig. 13 left). In contrast, our hierarchical approach can generate a layout that completely covers the whole domain. Third, we 


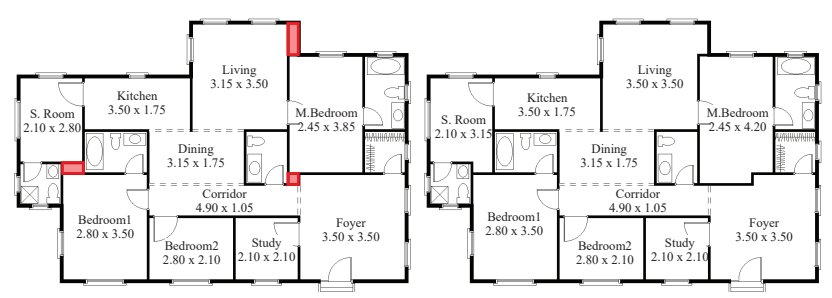

Figure 13: Given the same input, we perform a comparison between our basic method and our hierarchical approach. Left: the layout generated by our basic method. Regions that are not covered are highlighted in red. Right: the layout generated by the hierarchical approach.

\begin{tabular}{|l|lllc|}
\hline \multicolumn{1}{|c|}{ Figure } & \#Room & \#Constraints & Total & Time (sec.) \\
\hline Fig. 1 (a) & 13 & $13,13,2,1,3,8$ & 40 & 7.26 \\
Fig. 1 (b) & 154 & $154,154,0,0,0,0$ & 308 & 85.69 \\
Fig. 2 & 5 & $5,5,0,0,1,4$ & 15 & 0.13 \\
Fig. 4 (a) & 5 & $5,5,0,1,3,4$ & 18 & 0.16 \\
Fig. 4 (b) & 15 & $15,15,2,1,7,14$ & 54 & 11.31 \\
Fig. 7 & 14 & $14,14,1,1,6,13$ & 49 & 18.17 \\
Fig. 8 & 140 & $140,140,0,6,0,0$ & 286 & 78.63 \\
Fig. 9 left & 15 & $15,15,2,1,7,14$ & 54 & 16.17 \\
Fig. 9 right & 15 & $15,15,2,1,7,14$ & 54 & 11.09 \\
Fig. 10 & 15 & $15,15,2,1,7,14$ & 54 & 17.95 \\
Fig. 11 & 13 & $13,13,2,1,2,6$ & 37 & 1.52 \\
Fig. 12 & 13 & $13,13,2,1,5,11$ & 45 & 10.44 \\
Fig. 13 left & 15 & $15,15,2,1,7,14$ & 54 & 10.69 \\
Fig. 13 right & 15 & $15,15,2,1,7,14$ & 54 & 10.72 \\
Fig. 15 left & 119 & $119,119,0,6,0,0$ & 244 & 67.14 \\
Fig. 15 right & 95 & $95,95,0,0,0,0$ & 190 & 53.57 \\
Fig. 16 left & 111 & $111,111,0,1,0,0$ & 223 & 61.75 \\
Fig. 16 right & 154 & $154,154,0,0,0,0$ & 208 & 86.88 \\
\hline
\end{tabular}

Table 1: For each example shown in the paper, we present the number of rooms, the number of each kind of constraint, the number of total constraints, and running time. In the third column, the numbers of $C_{\text {size }}, C_{\text {overlap }}, C_{\text {ratio }}, C_{\text {pos }}, C_{\text {boundary }}$, and $C_{a d j}$ constraints are given.

evaluate the effect of each constraint by performing leave-one-out tests. Fig. 14 shows the effect of each constraint. We can see that the kitchen size is unreasonable if the size error term is excluded. Without the aspect ratio constraint, the kitchen is too narrow. The foyer is not connected to the main door without the position constraint. When the boundary constraint for the kitchen is excluded, the kitchen is not adjacent to the boundary of the house, which is not good for emission of kitchen exhaust. When the room adjacency constraints are excluded, there is no pathway to other rooms except the study. The performance of our algorithm is shown in Table 1 . In our experiments, our algorithm can generate a mid-size layout in 15 seconds on average.

We also evaluate our method on large-scale examples, e.g., rooms in an office building, booths in a shopping mall, and shelves in a supermarket. Fig. 15 shows two layouts of office buildings generated by our algorithm. In Fig. 16, two layouts of supermarkets are shown.

Comparison with other methods. For comparison, we implemented a stochastic optimization algorithm, i.e., the non-parallel version of [MSK10]. In the initialization step, we add rectangular rooms with the same size to the layout domain. The approach iteratively performs one of the following operations randomly: (1) swapping rooms or (2) sliding a wall. We evaluate the proposed layout by the objective value $E$. If $E$ is smaller than the objective value of previous layout $E$, we always accept the layout; otherwise, we accept it with the probability of $\exp \left(-E^{\prime}-E\right) / t$, where $t$ is the temperature. We gradually reduce the temperature $t$ in each iteration. The algorithm terminates when $E$ is smaller than a threshold. Fig. 18 shows the performance of the two methods on different complexities of the problem, where the complexity of the problem is defined as the sum of the number of rooms and the number of specified constraints. As the complexity of the input increases, the running time of the stochastic method increases significantly, while the running time of our method increases slowly. When the sum of the number of rooms and the number of constraints is larger than 50, the stochastic method takes more than two hours. To illustrate the problem, Fig. 17 left shows a layout generated by our algorithm, while Fig. 17 right shows a layout generated by the stochastic method that took 10 times longer to generate than our method took. At this point in time, the stochastic method has still not found a feasible solution that fulfills all constraints. We find that there is an uncovered region and several rooms are too small for furniture.

Limitations. The main limitation of our method is that we cannot currently handle non axis-aligned polygons. One possible approach to overcome this limitation is to apply shape deformations to generate rooms in arbitrary shapes.

\section{Conclusions}

In this paper, we propose a novel method for interior layout design. We formulate the problem using mixed integer quadratic programming. We propose a parametric layout representation suitable for this optimization framework and derive how to model five important high-level constraints for layout modeling as linear constraints. We propose a hierarchical framework to generate complicated layouts in a coarse-to-fine manner. We demonstrate that our method is faster by multiple orders of magnitude than previous work using stochastic optimization. Our work can generate a wide variety of layouts, such as residential buildings, exhibitions, supermarkets, department stores, etc. In future work, we would like to extend our method to other layout design problems, e.g., warehouses, game layouts, and electrical layouts. We also would like to extend our hierarchical framework to large-scale urban planning. It would also be interesting to integrate the idea of network design to generate layouts that satisfy both shape and network constraints.

\section{Acknowledgment}

This work was supported by the KAUST Office of Sponsored Research (OSR) under Award No. OCRF-2014-CGR3-62140401, and 


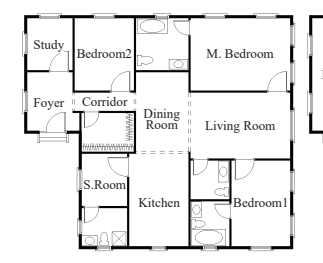

(a) all included

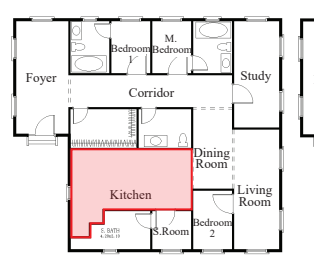

(b) size error term excluded

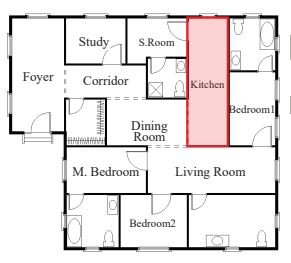

(c) aspect ratio constraint excluded

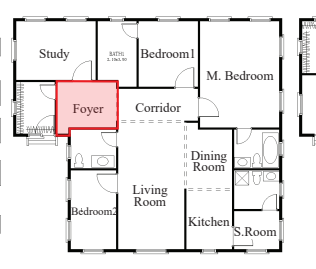

(d) position constraint excluded

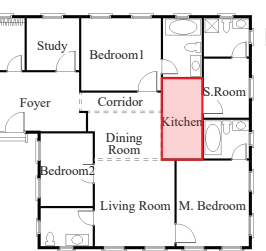

(e) boundary constraint exclude

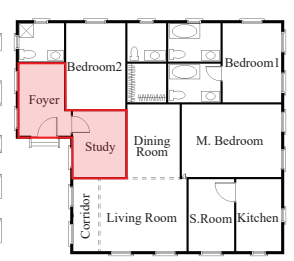

(f) adjacency constraint excluded

Figure 14: The effect of each constraints. (a) We extract the attributes from a real layout design. (b-f) Ablation of individual constraints and the effect on the results. The unreasonable part of each layout is highlighted.

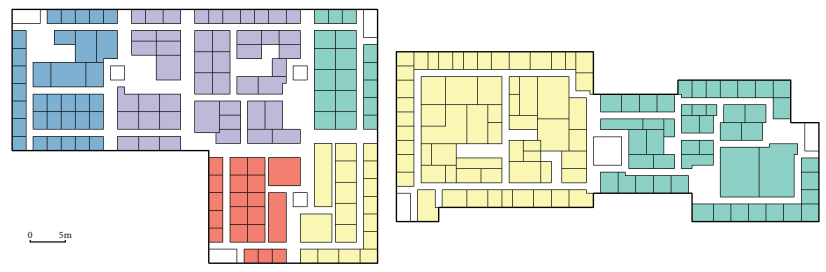

Figure 15: Interior layouts of office buildings generated by our method. Polygons in the same color are from the same parent rectangle in a higher level.
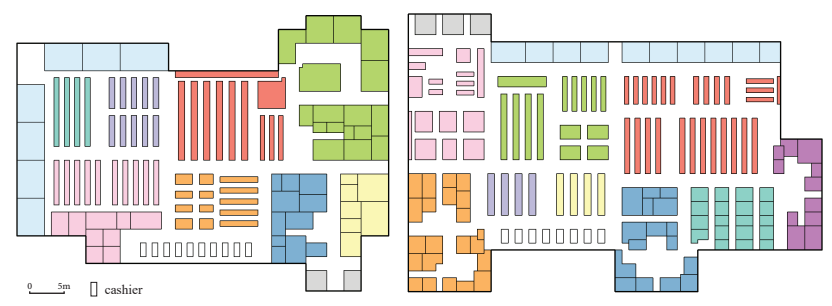

Figure 16: Interior layouts of supermarkets generated by our method. Shelves for products in the same category are in the same color.
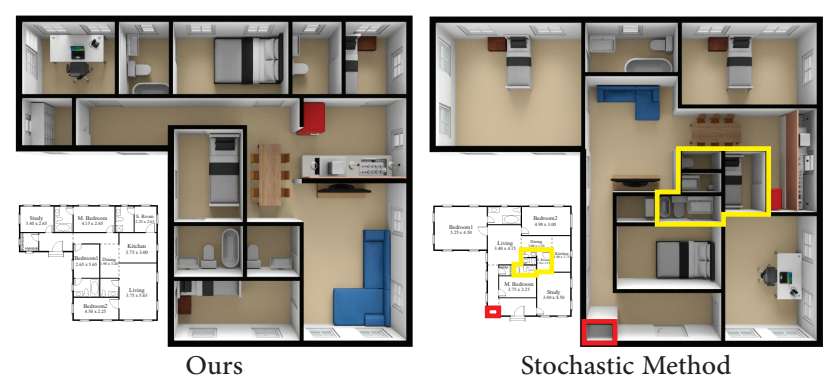

Figure 17: Given the same input (i.e., the layout domain and constraints), layouts generated by our method and the stochastic method are shown. The stochastic method took 10 times longer than our method. The empty region is highlighted in red, and rooms that are too small for furniture are highlighted in yellow.

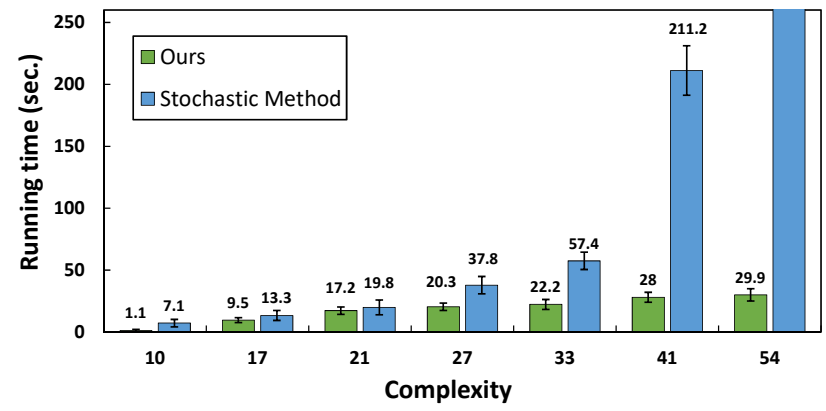

Figure 18: Performance comparison between the stochastic method and our algorithm. For the stochastic method, the running time increases significantly when the complexity (i.e., the sum of the number of rooms and the number of constraints) of the problem increases, while the running time of our approach increases slowly. The running time of the stochastic method for the last experiment is longer than 2 hours. We terminated it before obtaining a result.

the Visual Computing Center at KAUST. Ligang Liu is supported by the National Natural Science Foundation of China (61672482, 61672481, 11626253) and the One Hundred Talent Project of the Chinese Academy of Sciences. We would like to thank Virginia Unkefer for proofreading the paper.

\section{References}

[Ali12] Aliaga D. G.: 3D design and modeling of smart cities from a computer graphics perspective. ISRN Computer Graphics 2012 (2012). 3

[AVB08] Aliaga D. G., Vanegas C. A., Benes B.: Interactive example-based urban layout synthesis. In ACM transactions on graphics (TOG) (2008), vol. 27, ACM, p. 160. 3

[BBM*17] Bahrehmand A., Batard T., Marques R., Evans A., BLAT J.: Optimizing layout using spatial quality metrics and user preferences. Graphical Models (2017). 2

[BF91] BAYKAN C. A., FOX M. S.: Constraint satisfaction techniques for spatial planning. In Intelligent CAD Systems III. Springer, 1991, pp. 187-204. 2

[BYMW] BAO F., YAN D.-M., MitRa N. J., WONKA P.: Generating and exploring good building layouts. 3

[CC06] Chen T.-C., Chang Y.-W.: Modern floorplanning based on $\mathrm{b}^{*}$-tree and fast simulated annealing. IEEE Transactions on Computer- 
Aided Design of Integrated Circuits and Systems 25, 4 (2006), 637-650.

[CEW*08] Chen G., Esch G., Wonka P., Müller P., Zhang E. Interactive procedural street modeling. In ACM transactions on graphics (TOG) (2008), vol. 27, ACM, p. 103. 3

[CGC10] Chen G., Guo W., Chen Y.: A pso-based intelligent decision algorithm for vlsi floorplanning. Soft Computing 14, 12 (2010), 1329_ 1337. 2

[CZ10] CHEN J., ZHU W.: A hybrid genetic algorithm for vlsi floorplanning. In Intelligent Computing and Intelligent Systems (ICIS), 2010 IEEE International Conference on (2010), vol. 2, IEEE, pp. 128-132. 2

[DB08] Donath D., BöHmE L. F. G.: Constraint-based design in participatory housing planning. International Journal of architectural computing 6, 1 (2008), 97-117. 2

[Dou07] DoulgERAKIS A.: Genetic and embryology in layout planning. Master of Science in Adaptive Architecture and Computation, University of London (2007). 2

[DRCMPI07] Del Río-Cidoncha G., Martínez-Palacios J., IGLESIAS J. E.: A multidisciplinary model for floorplan design. International journal of production research 45, 15 (2007), 3457-3476.

[Dua05] DUARTE J. P.: A discursive grammar for customizing mass housing: the case of siza's houses at malagueira. Automation in construction 14, 2 (2005), 265-275. 2

[DZH*02] Dong S., Zhou S., Hong X., Cheng C., Gu J., CAI Y An optimum placement search algorithm based on extended corner block list. Journal of Computer Science and Technology 17, 6 (2002), 699-707.

[EF99] Elezkurtaj T., Franck G.: Genetic algorithms in support of creative architectural design. EUROPEAN COMPUTER AIDED AR CHITECTURAL DESIGN AND EDUCATION 17 (1999), 645-651. 2

[FUC* 88] Flemming, UlRich, Coyne, Glavin R. F., Timothy J.: A generative expert system for the design of building layouts - version 2. Cad and Robotics in Architecture and Construction (1988), 75-81. 2

[FYY*16] FENG T., YU L.-F., YEUNG S.-K., YIN K., ZHOU K.: Crowd-driven mid-scale layout design. ACM Trans. Graph. 35, 4 (2016), 132-1. 3

[GO16] GUROBi OptimizATION I.: Gurobi optimizer reference manual, 2016. URL: http: / / www.gurobi.com. 7

[Hsu00] HsU Y.-C.: Constraint based space planning: A case study. ACADIA Quarterly 19, 3 (2000), 2-3. 2

[Hua16] HUA H.: Irregular architectural layout synthesis with graphical inputs. Automation in Construction 72 (2016), 388-396. 3

[HWB95] HARADA M., WITKIN A., BARAFF D.: Interactive physically-based manipulation of discrete/continuous models. In Proceedings of the 22nd annual conference on Computer graphics and interactive techniques (1995), ACM, pp. 199-208. 2

[KF00] KIYOTA K., FUjIYOSHI K.: Simulated annealing search through general structure floorplans using sequence-pair. In Circuits and Systems, 2000. Proceedings. ISCAS 2000 Geneva. The 2000 IEEE International Symposium on (2000), vol. 3, IEEE, pp. 77-80. 2

[KS05] KeATRUANGKAMALA K., SINAPIROMSARAM K.: Optimizing architectural layout via mixed integer programming. In $C A A D$ FUTURES (2005), vol. 11, pp. 175-184. 2

[LC04] LIN J.-M., CHANG Y.-W.: Tcg-s: orthogonal coupling of $\mathrm{p} / \mathrm{sup} * /$-admissible representations for general floorplans. IEEE Transactions on Computer-Aided Design of Integrated Circuits and Systems 23, 6 (2004), 968-980. 2

[LFT00] Li S.-P., FrAZER J., TANG M.-X.: A constraint based generative system for floor layouts. 2

[LHP11] Leblanc L., Houle J., Poulin P.: Component-based modeling of complete buildings. In Proceedings of Graphics Interface 2011
(2011), Canadian Human-Computer Communications Society, pp. $87-$ 94. 2

[LWKF17] LIU C., WU J., Kohli P., FuRUKAWA Y.: Raster-to-vector: Revisiting floorplan transformation. In International Conference on Computer Vision (ICCV) (2017). 3

[LYAM13] LiU H., Yang Y.-L., AlHalawani S., Mitra N. J. Constraint-aware interior layout exploration for pre-cast concrete-based buildings. The Visual Computer 29, 6-8 (2013), 663-673. 3

[MAR07] MONi D. J., ARUMUGAM S., RANi G. N.: Vlsi floorplanning relying on differential evolution algorithm. ICGST International Journal on Artificial Intelligence and Machine Learning 7, 1 (2007), 62-67. 2

[MSK10] Merrell P., SchKufza E., Koltun V.: Computergenerated residential building layouts. In ACM Transactions on Graphics (TOG) (2010), vol. 29, ACM, p. 181. 1, 3, 9

[MVLS14] Ma C., Vining N., Lefebvre S., Sheffer A.: Game level layout from design specification. In Computer Graphics Forum (2014), vol. 33, Wiley Online Library, pp. 95-104. 3

[MXM09] MAO F., XU N., MA Y.: Hybrid algorithm for floorplanning using $\mathrm{b}^{*}$-tree representation. In Intelligent Information Technology Application, 2009. IITA 2009. Third International Symposium on (2009), vol. 3, IEEE, pp. 228-231. 2

[NFMK97] Nakatake S., Fujiyoshi K., Murata H., Kajitani Y. Module placement on bsg-structure and ic layout applications. In Proceedings of the 1996 IEEE/ACM international conference on Computeraided design (1997), IEEE Computer Society, pp. 484-491. 2

[Nil06] NiLKAEW P.: Assistant tool for architectural layout design by genetic algorithm. 2

[NNA06] NinOmiYa H., Numayama K., Asai H.: Two-staged tabu search for floorplan problem using o-tree representation. In Evolutionary Computation, 2006. CEC 2006. IEEE Congress on (2006), IEEE, pp. 718-724. 2

[PYB*16] PENG C.-H., YANG Y.-L., BAO F., Fink D., YAN D.-M., Wonka P., Mitra N. J.: Computational network design from functional specifications. ACM Transactions on Graphics (TOG) 35, 4 (2016), 131. 3

[PYW14] Peng C.-H., YAng Y.-L., Wonka P.: Computing layouts with deformable templates. ACM Transactions on Graphics (TOG) 33 4 (2014), 99. 2, 3

[RCMLS96] RaU-Chaplin A., MACKAY-LyOnS B., SPIERENBURG P.: The lahave house project: Towards an automated architectural design service. Cadex 96 (1996), 24-31. 2

[RSM17] Rosser J. F., Smith G., Morley J. G.: Data-driven estimation of building interior plans. International Journal of Geographical Information Science 31, 8 (2017), 1652-1674. 3

[SDD12] Singha T., DuTta H., DE M.: Optimization of floor-planning using genetic algorithm. Procedia Technology 4 (2012), 825-829. 2

[Sec12] SECHEN C.: VLSI placement and global routing using simulated annealing, vol. 54. Springer Science \& Business Media, 2012. 2

[STBB14] SMelik R. M., Tutenel T., BidarRa R., Benes B.: A survey on procedural modelling for virtual worlds. In Computer Graphics Forum (2014), vol. 33, Wiley Online Library, pp. 31-50. 3

[SVW*11] SEngupta D., Veneris A., Wilton S., IVANOV A., SALEH R.: Sequence pair based voltage island floorplanning. In Green Computing Conference and Workshops (IGCC), 2011 International (2011), IEEE, pp. 1-6. 2

[YWVW13] YANG Y.-L., WANG J., VOUGA E., WonKa P.: Urban pattern: Layout design by hierarchical domain splitting. ACM Transactions on Graphics (TOG) 32, 6 (2013), 181. 3 